\title{
Mechanoelectrical Feedback: Independent Role of Preload and Contractility in Modulation of Canine Ventricular Excitability
}

\author{
Bruce B. Lerman, Daniel Burkhoff, David T. Yue, and Kiichi Sagawa \\ Cardiology Division, Department of Medicine and Department of Biomedical Engineering, \\ The Johns Hopkins Medical Institutions, Baltimore, Maryland 21205
}

\begin{abstract}
Mechanoelectrical feedback, defined as changes in mechanical state that precede and alter transmembrane potential, may have potential importance in understanding the role of altered load and contractility in the initiation and modulation of ventricular arrhythmias. To assess the independent effects of preload and contractility on myocardial excitability and action potential duration, we determined the stimulus strength-interval relationship and recorded monophasic action potentials in isolated canine left ventricles contracting isovolumically. The strength-interval relationship was characterized by three parameters: threshold excitability, relative refractory period, and absolute refractory period. The effects of a threefold increase in left ventricular volume or twofold increase in contractility on these parameters were independently assessed. An increase in preload did not change threshold excitability in 11 ventricles but significantly shortened the absolute refractory period from $205 \pm 15$ to $191 \pm 14 \mathrm{~ms}$ ( $P$ $<0.001)$ (mean \pm SD). Similarly, the relative refractory period decreased from $220 \pm 18$ to $208 \pm 19 \mathrm{~ms}(P<0.002)$. Comparable results were observed when contractility was increased as a result of dobutamine infusion in 10 ventricles. That is, threshold excitability was unchanged but the absolute refractory period decreased from $206 \pm 14$ to $181 \pm 9 \mathrm{~ms}(P<0.003)$, and the relative refractory period decreased from $225 \pm 17$ to $205 \pm 18 \mathrm{~ms}(P$ $<0.003)$. Similar results were obtained when contractility was increased with $\mathrm{CaCl}_{2}$, indicating that contractility associated changes were independent of $\beta$-adrenergic receptor stimulation. An increase in preload or contractility was associated with shortening of the action potential. A threefold increase in preload and twofold increase in contractility were associated with a decrease in action potential duration of 22 and $24 \mathrm{~ms}$, respectively. There was a significant linear correlation between action potential duration and excitability (absolute refractory period). The similar effects of increased preload and contractility on threshold excitability and refractoriness can be explained by the action these perturbations have on the time course of repolarization. Therefore, excitability of the ventricle is sensitive to and is modulated by alteration of load or inotropic state. The similar effects of either increased preload or contractility on excitability may be
\end{abstract}

This work was presented in part at the Young Investigator's Awards Finalists Competition of the American College of Cardiology, March 1985, Anaheim, CA.

Dr. Lerman's present address is Cardiology Division, Box 158, University of Virginia School of Medicine, Charlottesville, VA 22908.

Received for publication 23 October 1984 and in revised form $15 \mathrm{July}$ 1985.

J. Clin. Invest.

(c) The American Society for Clinical Investigation, Inc.

0021-9738/85/11/1843/08 $\$ 1.00$

Volume 76, November 1985, 1843-1850 mediated by a common cellular mechanism which results in a rise in intracellular free $\mathrm{Ca}^{2+}$ and secondary abbreviation of the action potential.

\section{Introduction}

Left ventricular dysfunction has been identified as the strongest independent predictor of sudden death in patients with ventricular tachyarrhythmias (1). Despite the clinical recognition that depressed contractility and altered loading conditions can result in heart failure and precipitate ventricular fibrillation (classified as "secondary" ventricular fibrillation), the significance of mechanoelectrical feedback and its role in arrhythmogenesis has received minimal attention $(2,3)$. Mechanoelectrical feedback, defined as changes in mechanical state that precede and alter transmembrane potential, has been primarily investigated in isolated muscle preparations or in nonmammalian hearts.

The purpose of this study was to characterize the independent contributions of preload and contractility on electrical excitability and action potential duration in the intact ventricle. We used an isolated, cross-circulated canine left ventricle. This preparation permits precise measurement of pressure and volume as well as independent modification of preload and contractility (4-6). This model also circumvents reflex changes in contractility caused by changes in load and allows maintenance of a constant coronary artery perfusion pressure.

\section{Methods}

\section{Preparation}

The methods used to isolate and support the canine heart were similar to those previously published (7). Briefly, 15 pairs of mongrel dogs weighing 18-24 kg were anesthetized with sodium pentobarbital $(30 \mathrm{mg} /$ $\mathrm{kg}$, i.v.). A median sternotomy was performed under artificial ventilation on the donor dog. The left subclavian artery and right atrium were cannulated and connected to the femoral arteries and veins, respectively, of the support $\operatorname{dog}$ (Fig. 1). The brachiocephalic artery was cannulated for measurement of coronary perfusion pressure. The azygous vein, superior and inferior venae cavae, and descending aorta were ligated. Isolation was completed with ligation of the pulmonary hili at which time crosscirculation was begun. The heart was removed from the chest and suspended above a funnel. The left and right ventricles were vented. Coronary venous return drained into the funnel and was returned to the femoral vein of the support dog.

A thin latex balloon mounted on a metal adaptor was sewn to the mitral valve annulus. The balloon adaptor was then connected to a ventricular volume control servopump system (8) (Fig. 1). A constant volume of tap water filled the pump and balloon ( $55 \mathrm{ml}$, unstressed volume). The servopump was composed of a linear motor (model 411 , Ling Electronics, Anaheim, CA) that controlled the piston position of a rollingdiaphragm cylinder (model SS-4-F-SM, Bellofram Corp., Burlington, MA). A linear displacement transducer (model 240-000, Trans-Tek Corp., Ellington, CT) sensed the position of the piston, producing a signal proportional to the balloon volume. This signal was used in a negative feedback loop for comparison with a volume command signal that represented 


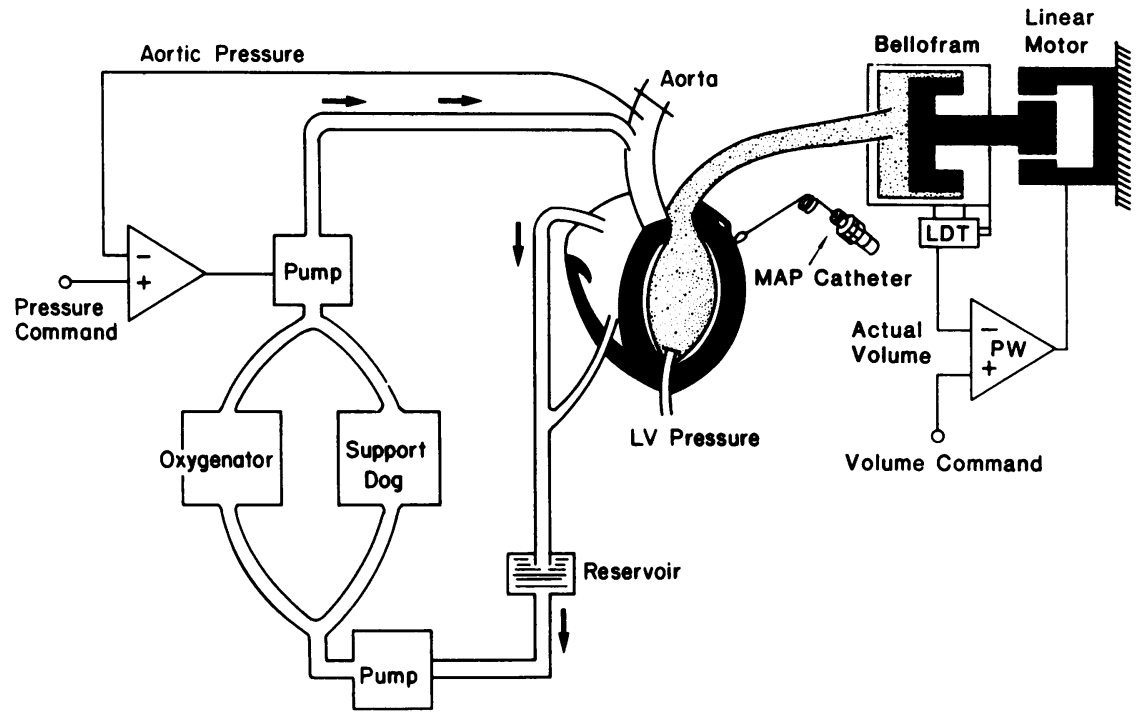

Figure 1. Schematic diagram of the preparation. The isolated left ventricle was perfused retrogradely from the left subclavian artery with blood from a support dog. The ventricular volume was controlled by a servopump system. A servopump also maintained coronary arterial pressure constant at $80 \mathrm{mmHg}$. A MAP electrode catheter was suspended by a spring-loaded system. LDT, linear displacement transducer; $L V$, left ventricle; $P W$, power amplifier.

the desired instantaneous volume. The error signal resulting from this comparison was supplied to a power amplifier (model DC-300, Crown International Co., Elkhart, IN) which then drove the linear motor.

To produce ejecting beats, an impedance loading system was developed that imposed a simulated arterial hydraulic impedance on the ventricle (8).

Isovolumic contractions were produced by inactivating the analogue integrator of the impedance loading system so that the volume command output was constant. A computer then directly controls the volume command signal through a digital-to-analogue converter. This system permitted accurate control and measurement of ventricular volume. The contractile state of the ventricle was quantified using concepts that have been developed in this laboratory (5). The end-systolic pressure-volume relationship (ESPVR) ${ }^{1}$ is linear for a given contractile state. The slope of this linear relationship, $E_{\max }$, has been shown to be insensitive to load but sensitive to changes in inotropic state and therefore has been used as an index of contractility. An increase in contractility (catecholamine infusion) is characterized by an increased slope of the pressure-volume regression line. The relationship between end-systolic pressure $\left(P_{e s}\right)$ and end-systolic volume $\left(V_{e s}\right)$ can be expressed by the following equation (6): $P_{\mathrm{es}}=E_{\max }\left[V_{\mathrm{es}}-V_{0}\right] . V_{0}$ is the volume axis intercept and represents the volume at which the ventricle cannot develop any transmural pressure. $V_{0}$ is invariant to changes in pressure or volume. Therefore, at a given end-systolic volume, $P_{e s}$ can be used as an index of contractility in an isovolumically beating heart.

Coronary arterial pressure was measured with a catheter placed in the aortic root. A servo-controlled perfusion pump (model 1215, Harvard Apparatus Co., Natick, MA) maintained mean coronary arterial pressure at $80 \mathrm{mmHg}$. Coronary arterial blood temperature was maintained at $37^{\circ} \mathrm{C}$ with a water bath and heat exchanger. The ESPVR was monitored on-line on a storage oscilloscope (model 5103, Tektronix, Inc., Beaverton, OR) and photographed with a Polaroid camera (Polaroid Corp., Cambridge, MA).

Coronary arterial pressure, left ventricular pressure and volume, $\mathrm{d} P$ / $\mathrm{d} t$, a modified electrocardiogram lead, bipolar myocardial electrogram (filtered at 30-500 Hz), contact epicardial monophasic action potential (MAP) (filtered DC-3 kHz), and the support dog's arterial pressure were recorded (model 2800, Gould, Inc., Cleveland, $\mathrm{OH}$ ). Signals from the MAP electrode were digitized at a sampling rate of $200 \mathrm{~Hz}$ by a PDP 11-23 computer (Digital Equipment Corp., Marlboro, MA).

1. Abbreviations used in this paper: ARP, absolute refractory period; ESPVR, end-systolic pressure-volume relationship; MAP, monophasic action potential; MAPD 90 , monophasic action potential duration at $90 \%$ repolarization; RRP, relative refractory period.
MAPs were recorded from the epicardial surface of the ventricle using a newly designed contact electrode (9). The recording device consists of an L-shaped cantilever with two electrodes (sintered $\mathrm{Ag}-\mathrm{AgCl}$ ) mounted on its distal end. One electrode forms the tip of the cantilever, which is pressed against the epicardium by a springloading mechanism. The other electrode is positioned $5 \mathrm{~mm}$ away from the tip. Electrical contact between this electrode and the heart is made through a small saline-soaked piece of foam rubber. This bipolar electrode arrangement in conjunction with differential amplification minimizes the effects of remote electrical activity on the MAP. The probe provided continuous MAP recordings of stable amplitude, smooth contour, and isopotential diastolic base lines for approximately a 2-h period allowing recordings to be made from a single epicardial site throughout the experimental protocol. Recordings from this electrode catheter have been shown to reflect the time course of transmembrane depolarization and repolarization (10).

Electrical excitability was quantified by the stimulus strength-interval relationship (11-13). Excitability has been shown to be an important factor in determining conduction velocity $(14,15)$. Excitability as determined in this study is dependent on the current density of the extracellular stimulating electrode, fiber orientation and intra- and intercellular excitability under the electrode (16-18).

The hearts were paced using unipolar cathodal stimulation with the stimulating electrode placed within $2 \mathrm{~mm}$ of the MAP and bipolar recording electrodes (midanterior wall). A digital computer controlled a constant current pacer that delivered rectangular pulses $2 \mathrm{~ms}$ in duration at twice diastolic threshold and was programmed to produce the pacing protocol used in the determination of strength-interval curves. This protocol consisted of an initial drive cycle of 10 stimuli $\left(S_{1}\right)$ at a cycle length of 500 or $600 \mathrm{~ms}$. This initial drive period was long enough to permit the ventricle to achieve a steady mechanical and electrical state (return to base line of the MAPD M0 , the MAP duration at $90 \%$ repolarization). A premature extrastimulus $\left(S_{2}\right)$ was introduced at a coupling interval $\left(S_{1}-S_{2}\right)$ of $250 \mathrm{~ms}$ with a stimulus strength equal to diastolic threshold. The coupling interval was decreased by 2 -ms intervals until there was failure to capture. The current of the extrastimulus was then increased by 0.1-mA steps until capture occurred. A 2-s pause followed the extrastimulus of each drive cycle. The above sequence was repeated until the stimulus strength of $S_{2}$ reached $10 \mathrm{~mA}$. The minimum current that resulted in consistent ventricular capture was defined as the diastolic threshold or threshold excitability. The longest coupling interval that required $>0.1-\mathrm{mA}$ increase in current for a $2-\mathrm{ms}$ decrement in coupling interval to elicit a depolarization was defined as the relative refractory period (RRP) and the absolute refractory period (ARP) was the longest coupling interval that failed to capture the ventricle at $10 \mathrm{~mA}$.

Cathodal stimulation was used instead of anodal or bipolar stimulation in order to avoid the complex ventricular response of supernormal 
excitability (11, 19). During bipolar threshold stimulation, excitation occurs at the cathode during diastole and at the anode during RRP. The latter response results in an early "diastolic dip" in the strength-interval curve that corresponds to supernormal excitability (13). The strengthinterval relationship, determined with cathodal current in our study, does not exhibit supernormal excitability.

\section{Experimental protocol}

Volume change. The left ventricle, contracting isovolumically, was paced at a rate of either 100 or 120 beats/min (i.e., cycle length of 600 and 500 $\mathrm{ms}$, respectively). After a stabilization period of 1-2 min, a control ESPVR was determined. The strength-interval relationship at a control volume ( 10 or $15 \mathrm{ml}$ ) and $\mathrm{MAPD}_{90}$ were then determined. The volume was then increased by 20 or $35 \mathrm{ml}$ (Fig. 2). $E_{\max }$ was determined by applying linear regression analysis to the ESPVR points obtained at the two volumes. After a stabilization period of $\sim 2 \mathrm{~min}$, the above pacing protocol sequence was repeated. The volume of the balloon was then reduced to the initial control value and the strength-interval relationship and MAPD $_{90}$ redetermined. Data were considered acceptable if results from the two control experiments were within $2 \%$ of each other.

To determine whether similar electrophysiologic effects were observed in ejecting hearts, a simulated arterial impedance on the left ventricle was imposed. The strength-interval relationship was determined at enddiastolic volumes of 25 and $50 \mathrm{ml}$, respectively, for two preparations.

Contractility study. At a volume of 10 or $15 \mathrm{ml}$ (identical volume as that used in the volume protocol for each particular dog) the strengthinterval relationship was determined and MAP recordings were obtained. Continuous infusion of dobutamine into the arterial perfusion line was then begun at a rate of $2-4 \mu \mathrm{g} / \mathrm{min}$. The infusion rate was adjusted to maintain the steady-state left ventricular end-systolic pressure at twice the control level (volume unchanged) (Fig. 3). The strength-interval relationship was then determined. Dobutamine infusion was then discontinued and the left ventricular end-systolic pressure allowed to return to control. After steady-state conditions were achieved, the strength-interval relationship was redetermined. The same criteria for acceptability of data used in the volume protocol were also used in this protocol.

To determine whether the alterations in excitability and MAPD $D_{90}$ related to changes in contractility were independent of $\beta$-adrenergic receptor stimulation, two experiments were performed in which $\mathrm{CaCl}_{2}$ was used to increase inotropy. This was infused into the donor heart at a rate of $0.1-0.4 \mathrm{~g} / \mathrm{min}(0.2 \mathrm{mM})$ in order to increase contractility twofold.

\section{Statistical analysis}

Statistical comparisons of intervention versus control were performed using Student's paired $t$ test. To determine whether a linear relationship existed between action potential duration and excitability, a linear regression based on the method of least squares was determined. All data are expressed as mean $\pm \mathrm{SD}$. Differences were considered significant for $P<0.05$.

\section{Results}

Effects of volume on the strength-interval relationship. An increase in left ventricular volume from 10 to $30 \mathrm{ml}$ or from 15 to $45 \mathrm{ml}$ did not alter threshold excitability, $0.8 \pm 0.3 \mathrm{~mA}$ (control) vs. $0.8 \pm 0.3 \mathrm{~mA}$ (increased volume) in 11 ventricles. In contrast, a significant change occurred in the ARP, $205 \pm 15 \mathrm{~ms}$ vs. $191 \pm 14$ ms, $P<0.001$. A decrease of similar magnitude was observed in the RRP, $220 \pm 18 \mathrm{~ms}$ vs. $208 \pm 19 \mathrm{~ms}, P<0.002$. A representative strength-interval relationship at the two different left ventricular volumes is illustrated in Fig. 4. As observed, the strengthinterval curve is nonlinear and closely resembles a decaying exponential relationship.

To determine whether increased volume had a similar effect on excitability for ejecting beats, a simulated arterial hydraulic impedance was imposed on the left ventricle. The strength-in- terval relationship was determined at end-diastolic volumes of 25 and $50 \mathrm{ml}$ for ejecting beats in two experimental preparations. Results were similar to those for isovolumic beats (Fig. 4).

Effects of contractility on the strength-interval relationship. In 10 dogs, volume was held constant at 10 or $15 \mathrm{ml}$ and the inotropic state was increased by a factor of 2 with dobutamine infusion. Results were similar to those obtained with increased preload. Threshold excitability was unchanged by enhanced contractility, $0.8 \pm 0.2 \mathrm{~mA}$ (control) vs. $0.7 \pm 0.2 \mathrm{~mA}$ (increased contractility). However, both the ARP and RRP decreased; ARP, $206 \pm 14$ ms vs. $181 \pm 9 \mathrm{~ms}, P<0.003$; RRP, $225 \pm 17 \mathrm{~ms}$ vs. $205 \pm 18 \mathrm{~ms}, P<0.003$. A representative strength-interval relationship for increased contractility is shown in Fig. 5. In two dogs, $\mathrm{CaCl}_{2}$ was infused instead of dobutamine in order to achieve a twofold increase in contractility. Effects on excitability were similar to those observed with dobutamine (Fig. 6). Though not quantified, spontaneous ventricular extrasystoles (pairs and triplets) were frequently observed following increases in preload and contractility.

Relationship between volume, contractility, and $M A P_{90}$. An increase in volume was associated with shortening of the MAPD $_{90}$ from $223 \pm 15 \mathrm{~ms}$ (control) to $211 \pm 17 \mathrm{~ms}$ (increased volume), $P<0.001$. Similar findings were observed with an increase in contractility. A twofold increase in $E_{\max }$ was accompanied by a decrease in MAPD $_{90}$ from $231 \pm 14 \mathrm{~ms}$ to $207 \pm 8$ ms, $P<0.001$.

Relationship between $M A P_{90}$ and excitability. To determine whether a linear correlation existed between MAPD M0 $_{90}$ and the ARP regression analysis was performed (Fig. 7). The change in MAPD $_{90}$ between control volume and augmented volume was compared to the change in ARP at these two volumes. In five experimental preparations, MAP recordings met stability and reproducibility criteria to permit inclusion. There was a significant linear correlation between $\Delta$ MAPD $_{90}$ and $\Delta \mathrm{ARP}, r=0.97$ ( $\Delta=$ difference between control and intervention). There was also a significant correlation between $\Delta$ MAPD $_{90}$ and $\Delta$ ARP for twofold changes in contractility, $r=0.92$.

\section{Discussion}

We have shown for the first time that preload and contractility each independently alter excitability of the mammalian ventricle as defined by the strength-interval relationship. These mechanical perturbations also shorten the time course of the action potential.

Lab and Kaufmann were among the first investigators to focus attention on the study of mechanoelectrical feedback ( 2 , 3). Extensive studies were performed primarily examining these interactions on a beat-to-beat basis $(3,20)$. In the present investigation, the effects of mechanical perturbations were studied after steady-state conditions were achieved rather than during single contractions, because it was thought to simulate the clinical analogue more closely.

Several groups of investigators have reported that stretch shortens action potential duration $(2,3,20,21)$ and induces extrasystoles and repetitive firing (22) in isolated muscle preparations and the frog ventricle. The relevance of these findings to the intact mammalian ventricle is unclear in that stretch in an anchored isolated muscle preparation does not provide the same stress-strain effects that increased preload does in an intact left ventricle. Furthermore, internal calcium kinetics in amphibian and mammalian hearts are significantly different (23). 

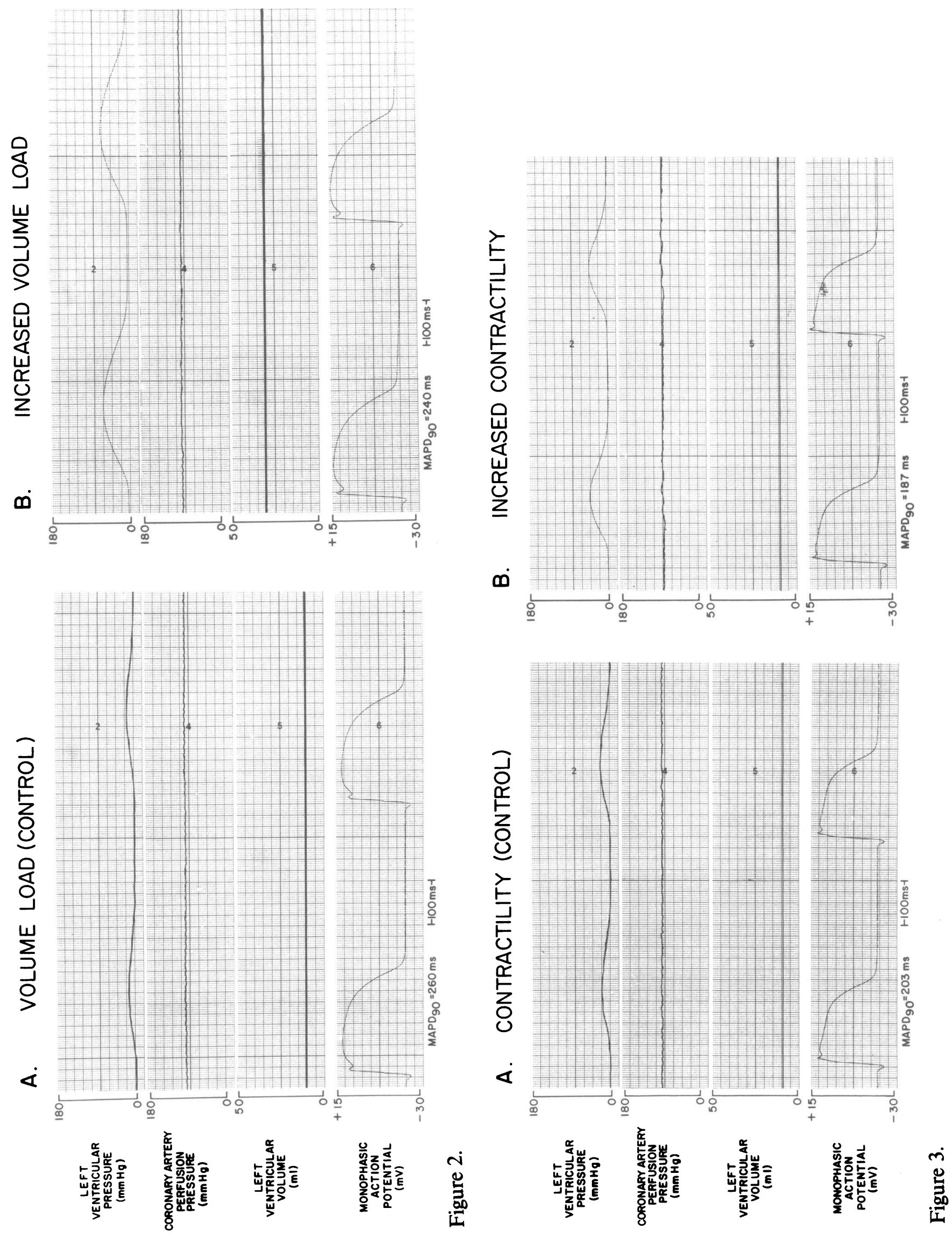

1846 Lerman et al. 


\section{A.}
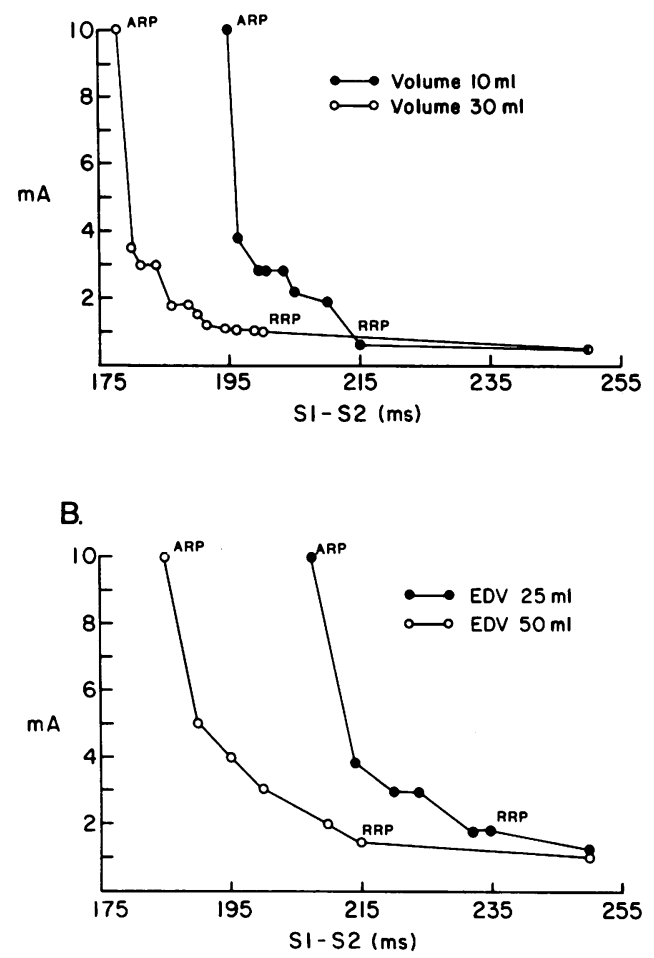

Figure 4. (A) Representative effect of volume on the strength-interval relationship in an isovolumically beating left ventricle. Coupling intervals of the extrastimulus $\left(S_{1}-S_{2}\right)$ are plotted versus the maximum milliamperage $(\mathrm{mA})$ failing to capture the ventricle at that interval. Threshold excitability was unchanged by the increase in volume while the RRP and ARP were decreased. $(B)$ Effect of volume on the strength-interval relationship in an ejecting left ventricle. Effects on threshold excitability, the RRP, and ARP were similar to those observed in isovolumically beating hearts. EDV, end diastolic volume.

Despite these qualifications, however, effects of stretch observed in these experimental preparations are qualitatively similar to our findings when preload was increased in the mammalian ventricle.

An increase in tension or contractility in isolated muscle has been shown to precede and alter action potential duration resulting in an inverse relationship between inotropic state and action potential duration (24-27). That is, an increase in contractility shortens the action potential, a relationship that we found in the intact left ventricle.

Implications for arrhythmogenesis. The observed changes in

Figure 2. $(A)$ Recording of left ventricular pressure, coronary artery perfusion pressure, left ventricular volume $(10 \mathrm{ml})$ and MAP. Volume tracing was retouched. $(B)$ Recording of the above parameters when the left ventricular volume was increased to $30 \mathrm{ml}$. The inotropic state (contractility) remained unchanged. Note that the MAPD 90 decreased by $20 \mathrm{~ms}$ at the higher volume. Volume tracing was retouched.

Figure 3. (A) Recordings of a representative contractility experiment (control). Top two tracings were retouched. $(B)$ Recording of measured parameters at twice the control peak left ventricular pressure (contractility). The MAPD ${ }_{90}$ decreased by $16 \mathrm{~ms}$. The preload volume remained constant throughout the experiment. Coronary artery perfusion pressure tracing was retouched. excitability related to mechanical perturbation may have important implications for understanding arrhythmogenesis under altered mechanical conditions. Brooks and associates (28) reported that ischemia caused electrophysiologic effects similar to those observed during the mechanical perturbations in our study; specifically, increased excitability and shortening of the action potential. They also noted that ventricular extrasystoles and fibrillation occurred spontaneously during the period of significant action potential shortening. We also observed a marked increase in spontaneous extrasystoles during action potential shortening and an increase in excitability after augmentation of load or inotropic state. A possible mechanism to explain how the mechanical effects we observed can initiate arrhythmias involves the generation of anodal current by premature repolarization to surrounding cells. This anodal current can accelerate repolarization of contiguous cells and disorganize the normal recovery sequence $(28,29)$. Anodal current can also stimulate proximate cells during their period of vulnerability (supernormal excitability) causing premature excitation and initiation of fibrillation (28).

Our findings may not only have relevance for understanding the initiation of arrhythmias but also for the potentiation of reentrant arrhythmias. Reentry depends on nonuniform recovery of excitability and repolarization (30-33). Repolarization in normal myocardium is heterogeneous in both the transverse (endo-epicardium) and longitudinal planes (epicardium: apexbase) (34-37). Any perturbation that accelerates the normal dispersion of repolarization and excitability will potentiate the likelihood for initiation and maintenance of reentry. Acute ischemia, chronic infarction, and mode of mechanical contraction can cause marked inhomogeneity in repolarization and excitability (38-40). Khatib and Lab (40) showed that shortening of action potential due to an abrupt increase in pacing rate shows a disparity in effect between the apex and base during isovolumic contractions compared to ejecting beats. Alteration of preload and/or contractility may possibly exaggerate the electrical heterogeneity that normally exists due to asymmetry of ventricular geometry and wall stress and thus potentiate the probability for developing reentrant arrhythmias.

Another potential mechanism of arrhythmogenesis that may explain, in part, the increased ventricular ectopy observed with augmented preload or contractility is mechanically induced afterdepolarizations that reach threshold potential and result in extrasystoles. Pressure overload of either the right or left ventricle has been shown to cause an inhomogeneous contraction pattern, i.e., lengthening instead of shortening during systole, that is associated with afterdepolarizations occurring during repolarization (phase 3) $(41,42)$.

The similar effects of increased preload and contractility on threshold excitability (no change) and those observed on the refractory periods (decrease) can be explained by the action these perturbations have on the time course of repolarization. The change in the ARP between control and intervention (increase in preload or contractility) was linearly correlated with the degree of shortening in MAP duration. Therefore, threshold excitability, determined near mid-diastole, would not have been expected to change in response to mechanical perturbation in that membrane potential would have had sufficient time to recover to its resting level at the time of the premature stimulus $\left(S_{2}\right)$. However, shortening of the MAP secondary to increased load or contractility permitted more rapid recovery of voltage-dependent excitability than that achieved during the control period. This allowed pre- 


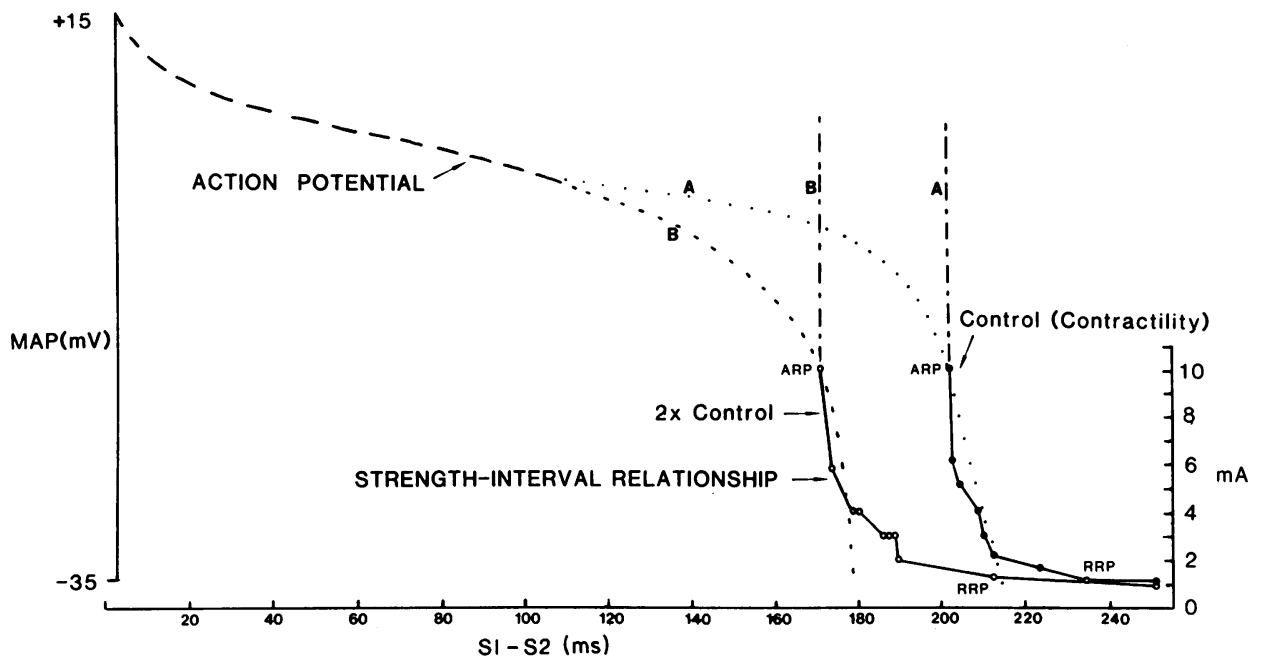

Figure 5. Effect of increased contractility (dobutamine infusion) on the strength-interval relationship from a representative experimental preparation. An increase in contractility had similar effects as volume on the threshold excitability, the RRP and ARP. Superimposed on the graphs are diagrammatic representations of the respective MAPs resulting from the two levels of inotropic state. Increased inotropy shortens the action potential duration $(B)$ and allows membrane potential to recover earlier than the action potential associated with the lower inotropic state $(A)$. Therefore, because enhanced inotropy accelerates repolarization, premature extrastimuli are able to excite the ventricle at a coupling interval that would find the ventricle refractory at a lower inotropic state. mature extrastimuli to excite the ventricle at a coupling interval that otherwise would have found the ventricle refractory at a lower preload or inotropic state (Fig. 5).

Cellular mechanisms. The findings that increase in preload and contractility each produce similar directional change in both excitability and action potential duration may appear unexpected, particularly if the inotropic state of cardiac muscle is considered independent of muscle length (43). However, diastolic volume and inotropic state may not be independent determinants of myocardial performance in that developed tension at a given muscle length is dependent on the degree of activation of the contractile system and conversely, activation of the contractile system depends on resting muscle length (length-dependent activation) $(44,45)$. Length-dependent activation has also been shown to be operative in the intact ventricle (46). Therefore, increased preload and contractility may exert their similar effects on excitability and action potential duration through a common cellular mechanism.

There are two primary explanations to account for the lengthdependent process. The first hypothesis suggests that the contractile apparatus is more sensitive to calcium at greater sarcomere lengths $(47,48)$. The other explanation suggests that lengthdependent activation results from an increase in intracellular

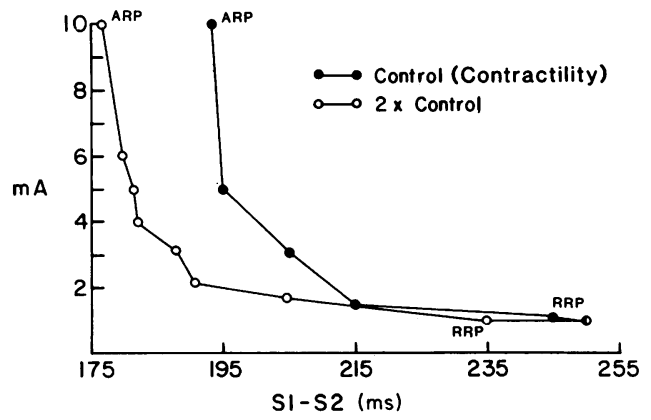

Figure 6. Effect of increased contractility produced by $\mathrm{CaCl}_{2}$ infusion on the strength-interval relationship. Effects were similar to those observed with dobutamine infusion at the same level of inotropy. free calcium $\left[\mathrm{Ca}^{2+}\right]_{\mathrm{i}}(45)$. Studies performed in skeletal muscle using the calcium-sensitive photoprotein aequorin indicate that an increase in resting length is associated with an increase in $\left[\mathrm{Ca}^{2+}\right]_{\mathrm{i}}(49,50)$. Length-dependent release of calcium from the
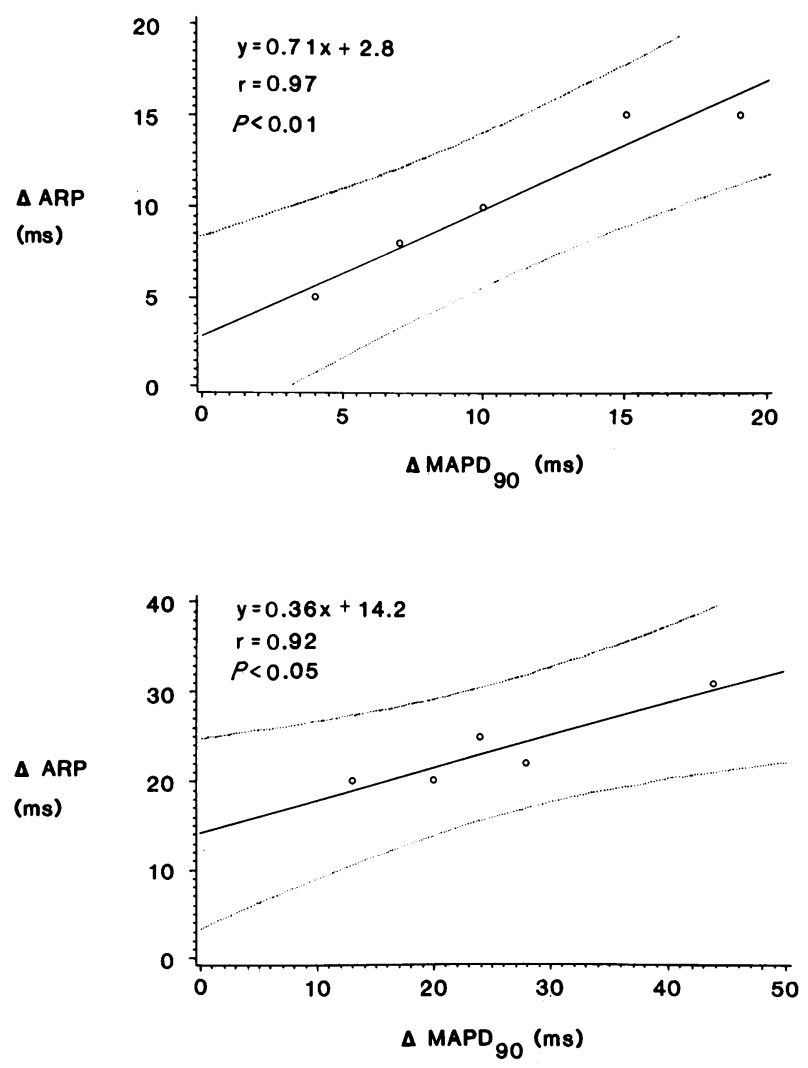

Figure 7. $(A)$ Linear regression between $\Delta \mathrm{MAPD}_{90}$ and $\triangle \mathrm{ARP}$ for volume experiments. $\Delta$ represents the difference between control and intervention value. Each data point represents results from one animal. Dotted lines represent the $95 \%$ confidence interval. (B) Linear regression between $\triangle M A P D_{90}$ and $\triangle A R P$ for contractility experiments. 
sarcoplasmic reticulum has also been demonstrated in skinned cardiac cells (51). In addition, elevation of $\left[\mathrm{Ca}^{2+}\right]_{i}$ may increase $\mathrm{K}^{+}$conductance (52-56) and decrease the electrochemical gradient of the slow inward current $\left(i_{\mathrm{si}}\right)(57)$. Therefore, either an increase in the outward current and/or the decrease in $i_{\mathrm{si}}$ can shorten action potential duration. These findings may explain the mechanism by which both preload and contractility shortened the action potential and thereby increased excitability as observed in our study.

Elevated intracellular calcium also activates a transient inward current $\left(i_{\mathrm{TI}}\right)(58)$ that can induce oscillatory potentials and triggered automaticity (59). The current flows through a nonselective cation channel that is nearly equally permeable to both $\mathrm{Na}^{+}$and $\mathrm{K}^{+}(60,61)$. Increased intracellular calcium can also activate an electrogenic $\mathrm{Na}-\mathrm{Ca}$ exchange (62) in which $\mathrm{Na}^{+}$is the inward current. However, these two mechanisms cannot account for electrophysiologic effects that we observed because they prolong action potential duration, and not decrease it. They can, however, explain the effects recorded when cardiac muscle is released during an isometric contraction (63).

Another mechanism that may contribute to abbreviation of the action potential is rate-dependent accumulation of extracellular potassium (64). This effect, if operative, was unlikely to account for shortening of the action potential in this study because pacing was performed at identical cycle lengths for each intervention in each experiment.

In summary, we have demonstrated that excitability (ARP and RRP) is sensitive to and is modulated by alteration of loading conditions and inotropic state of the left ventricle. The effects of these mechanical perturbations are parallel in that an increase in either preload or contractility increases excitability. This similar electrophysiologic response to different mechanical interventions may reflect a common cellular mechanism-one that results in a rise in $\left[\mathrm{Ca}^{2+}\right]_{i}$ and increased membrane permeability to $\mathrm{K}^{+}$. Further confirmation that this form of mechanoelectrical feedback may have an important role in the initiation and modulation of ventricular arrhythmias will be supported by demonstrating that mechanical perturbations increase the nonuniform dispersion of excitability.

\section{Acknowledgments}

This work was supported by grant HL-17655-07 (Specialized Center of Research in Ischemic Heart Disease) from the National Heart, Lung and Blood Institute and research grant R01-HL-14903 from the National Institutes of Health.

\section{References}

1. Swerdlow, C. D., R. A. Winkle, and J. W. Mason. 1983. Determinants of survival in patients with ventricular tachyarrhythmias. $N$. Engl. J. Med. 308:1436-1442.

2. Lab, M. J. 1982. Contraction-excitation feedback in myocardium. Physiological basis and clinical relevance. Circ. Res. 50:757-766.

3. Kaufmann, R., M. J. Lab, R. Hennekes, and H. Krause. 1971. Feedback interaction of mechanical and electrical events in the isolated ventricular myocardium (cat papillary muscle). Pflügers Arch. Eur. J. Physiol. 324:110-123.

4. Suga, H., K. Sagawa, and A. A. Shoukas. 1973. Load independence of the instantaneous pressure-volume ratio of the canine left ventricle and effects of epinephrine and heart rate on the ratio. Circ. Res. 32:314322.

5. Suga, H., and K. Sagawa. 1974. Instantaneous pressure-volume relationships and their ratio in the excised, supported canine left ventricle. Circ. Res. 35:117-126.
6. Sagawa, K. 1978. The ventricular pressure-volume diagram revisited. Circ. Res. 43:677-687.

7. Suga, H., and K. Sagawa. 1977. End-diastolic and end-systolic ventricular volume clamper for isolated canine heart. Am. J. Physiol. 233:H718-H722.

8. Sunagawa, K., K. O. Lim, D. Burkhoff, and K. Sagawa. 1982. Microprocessor control of a ventricular volume servo-pump. Ann. Biomed. Eng. 10:145-159.

9. Franz, M. R., J. T. Flaherty, E. V. Platia, B. H. Bulkley, and M. L. Weisfeldt. 1984. Localization of regional myocardial ischemia by recording of monophasic action potentials. Circulation. 69:593-604.

10. Franz, M. R., D. Burkhoff, M. L. Weisfeldt, and E. G. Lakatta. 1984. Quantitative comparison of monophasic action potentials recorded simultaneously with an intra- and a new extracellular electrode. J. Am. Coll. Cardiol. 3:498. (Abstr.)

11. Orias, O., C. M. Brooks, E. E. Suckling, J. L. Gilbert, and A. A. Siebens. 1950. Excitability of the mammalian left ventricle throughout the cardiac cycle. Am. J. Physiol. 163:272-282.

12. van Dam, R. T., D. Durrer, J. Strackee, and L. H. van der Tweel. 1956. Excitability cycle of the dog's left ventricle determined by anodal, cathodal, and bipolar stimulation. Circ. Res. 4:196-204.

13. Brooks, C. M., B. F. Hoffman, E. E. Suckling, and O. Orias. 1955. Methods of studying cardiac excitability. In Excitability of the Heart. Grune \& Stratton, New York. 48-97.

14. Spear, J. F., and E. N. Moore. 1974. Supernormal excitability and conduction in the His-Purkinje system of the dog. Circ. Res. 35: 782-792.

15. Peon, J., G. R. Ferrier, and G. K. Moe. 1978. The relationship of excitability to conduction velocity in canine Purkinje tissue. Circ. Res. 43:125-135.

16. Fozzard, H. A., and M. Schoenberg. 1972. Strength-duration curves in cardiac Purkinje fibers. Effects of liminal length and charge distribution. J. Physiol. (Lond.). 226:593-618.

17. Dominguez, G., and H. A. Fozzard. 1970. Influence of extracellular $\mathrm{K}^{+}$concentration on cable properties and excitability of sheep cardiac Purkinje fibers. Circ. Res. 26:565-574.

18. Spach, M. S., W. T. Miller, D. B. Geselowitz, R. C. Barr, J. M. Kootsey, and E. A. Johnson. 1981. The discontinuous nature of propagation in normal canine cardiac muscle. Evidence for recurrent discontinuities of intracellular resistance that affect the membrane currents. Circ. Res. 48:39-54.

19. Cranefield, P. F., B. F. Hoffman, and A. A. Siebens. 1957. Anodal excitation of cardiac muscle. Am. J. Physiol. 190:383-390.

20. Lab, M. J. 1978. Mechanically dependent changes in action potentials recorded from the intact frog ventricle. Circ. Res. 42:519-528.

21. Dudel, J., and W. Trautwein. 1954. Das Aktionspotential and Mechanogramm des Herzmuskels unter dem Einflus der Dehnung. Cardiologie. 25:344-362.

22. Kaufmann, R., and U. Theophile. 1967. Automatie fordernde Dehnungseffects an Purkinje Faden, Pappilarmuskeln und Vorhoftrabekeln von Rheususaften. Pflügers Arch. Eur. J. Physiol. 291:174-189.

23. Morad, M., and Y. Goldman. 1973. Excitation-contraction coupling in heart muscle: membrane control of development of tension. Prog. Biophys. Mol. Biol. 27:257-313.

24. Greenspan, K., R. E. Edmunds, and C. Fisch. 1967. The relation of contractile enhancement to action potential change in canine myocardium. Circ. Res. 20:311-320.

25. Lu, H., G. Lange, and C. M. Brooks. 1968. Comparative studies of electrical and mechanical alternation in heart cells. J. Electrocardiol. 1:7-17.

26. Spear, J. F., and N. Moore. 1971. A comparison of alternation in myocardial action potentials and contractility. Am. J. Physiol. 220: 1708-1716.

27. Boyett, M. R. 1978. An analysis of the effect on the rate of stimulation and adrenaline on the duration of the cardiac action potential. Pflügers Arch. Eur. J. Physiol. 377:155-166.

28. Brooks, C. M., J. L. Gilbert, M. E. Greenspan, G. Lange, and 
H. M. Mazzella. 1960. Excitability and electrical response of ischemic heart muscle. Am. J. Physiol. 198:1143-7.

29. Cranefield, P. F., and B. F. Hoffman. 1958. Propagated repolarization in heart muscle. J. Gen. Physiol. 41:633-649.

30. Han, J., and G. K. Moe. 1964. Nonuniform recovery of excitability in ventricular muscle. Circ. Res. 14:44-60.

31. Merx, W., M. S. Yoon, and J. Han. 1977. The role of local disparity in conduction and recovery time on ventricular vulnerability to fibrillation. Am. Heart. J. 94:603-610.

32. Allessie, M. A., F. I. M. Bonke, and F. J. G. Schopman. 1976. Circus movement in rabbit atrial muscle as a mechanism of tachycardia. II. The role of nonuniform recovery of excitability in the occurrence of unidirectional block as studied with multiple microelectrodes. Circ. Res. 39:168-177.

33. Kuo, C. S., K. Munakata, P. Reddy, and B. Surawicz. 1983. Characteristics and possible mechanism of ventricular arrhythmia dependent on the dispersion of action potential durations. Circulation. 67: 1356-1367.

34. Moore, E. N., J. B. Preston, and G. K. Moe. 1965. Duration of transmembrane action potentials and functional refractory periods of canine false tendon and ventricular myocardium. Circ. Res. 17:259273.

35. Spach, M. S., and R. C. Barr. 1975. Ventricular intramural and epicardial potential distributions during ventricular activation and repolarization in the intact dog. Circ. Res. 37:243-257.

36. Burgess, M. J., L. S. Green, K. Millar, R. Wyatt, and J. A. Abildskov. 1972. The sequence of normal ventricular recovery. Am. Heart J. 84:660-669.

37. Autenrieth, G., B. Surawicz, and C. S. Kuo. 1975. Sequence of repolarization on the ventricular surface in the dog. Am. Heart J. 89: 463-469.

38. Downar, E., M. J. Janse, and D. Durrer. 1977. The effect of "ischemic" blood on transmembrane potentials of normal porcine ventricular myocardium. Circulation. 55:455-462.

39. Michelson, E. L., J. F. Spear, and E. N. Moore. 1980. Electrophysiologic and anatomic correlates of sustained ventricular tachyarrhythmias in a model of chronic myocardial infarction. Am. J. Cardiol. 45:583-590.

40. Khatib, S. Y., and M. J. Lab. 1981. Differences in electrical activity in the apex and base of left ventricle produced by exchanges in mechanical conditions of contraction. J. Physiol. (Lond.). 324:25P-26P.

41. Lab, M. J. 1978. Depolarization produced by mechanical changes in normal and abnormal myocardium. J. Physiol. (Lond.). 248:143P$144 \mathrm{P}$.

42. Covell, J. W., M. J. Lab, and R. Pavelec. 1981. Mechanical induction of paired action potentials in intact heart in situ. J. Physiol. (Lond.). 320:34P.

43. Anderson, P. A. W., A. Manring, and E. A. Johnson. 1973. Forcefrequency relationship - a basis for a new index of cardiac contractility? Circ. Res. 33:665-671.

44. Lakatta, E. G., and B. R. Jewell. 1977. Length-dependent activation; its effect on the length-tension relation in cat ventricular muscle. Circ. Res. 40:251-257.

45. Jewell, B. R. 1977. A reexamination of the influence of muscle length on myocardial performance. Circ. Res. 40:221-230.
46. Tucci, P. J. F., E. A. Bregagnollo, J. Spadaro, A. C. Cicogna, and M. C. L. Ribeiro. 1984. Length dependence of activation studied in the isovolumic blood-perfused dog heart. Circ. Res. 55:59-66.

47. Fabiato, A., and F. Fabiato. 1978. Myofilament-generated tension oscillations during partial calcium activation and activation dependence of the sarcomere length-tension relation of skinned cardiac cells. J. Gen. Physiol. 72:667-699.

48. Hibberd, M. G., and B. R. Jewell. 1982. Calcium and lengthdependent force production in rat ventricular muscle. J. Physiol. (Lond.). 329:527-539.

49. Ridgway, E. B., and A. M. Gordon. 1975. Muscle activation: effects of small length changes on calcium release in single fibers. Science (Wash. DC). 189:881-884.

50. Taylor, S. R., R. Rudel, and J. R. Blanks. 1975. Calcium transients in amphibian muscle. Fed. Proc. 34:1379-1381.

51. Fabiato, A., and F. Fabiato. 1975. Dependence on the contractile activation of skinned cardiac cells on the sarcomere length. Nature (Lond.). 256:54-56.

52. Isenberg, G. 1975. Is potassium conductance of cardiac Purkinje fibers controlled by $\left[\mathrm{Ca}^{2+}\right]_{\mathrm{i}}$ ? Nature (Lond.). 253:273-274.

53. Isenberg, G. 1977. Cardiac Purkinje fibers. Resting, action, and pacemaker potential under the influence of $\left[\mathrm{Ca}^{2+}\right]_{i}$ as modified by intracellular injection techniques. Pflügers Arch. Eur. J. Physiol. 371:51-59.

54. Isenberg, G. 1977. Cardiac Purkinje fibers. $\left[\mathrm{Ca}^{2+}\right]_{i}$ controls steady state potassium conductance. Pflügers Arch. Eur. J. Physiol. 371:71-76.

55. Isenberg, G. 1977. Cardiac Purkinje fibers. $\left[\mathrm{Ca}^{2+}\right]_{\mathrm{i}}$ controls the potassium permeability via the conductance components $g_{\mathrm{K} 1}$ and $\overline{\boldsymbol{g}}_{\mathbf{K} 2}$. Pflügers Arch. Eur. J. Physiol. 371:77-85.

56. Bassingthwaighte, J. B., C. H. Fry, and J. A. S. McGuigan. 1976. Relationship between internal calcium and outward current in mammalian ventricular muscle; a mechanism for the control of action potential duration? J. Physiol. (Lond.). 262:15-37.

57. Reuter, H. 1973. Time- and voltage-dependent contractile responses in mammalian cardiac muscle. Eur. J. Cardiol. 1:177-181.

58. Kass, R. S., W. J. Lederer, R. W. Tsien, and R. Weingart. 1978. Role of calcium ions in transient inward currents and aftercontractions induced by strophanthidin in cardiac Purkinje fibres. J. Physiol. (Lond.). 281:187-208.

59. Wit, A. L., and P. F. Cranefield. 1976. Triggered activity in cardiac muscle fibers of the Simian mitral valve. Circ. Res. 38:85-98.

60. Kass, R. S., R. W. Tsien, and R. Weingart. 1978. Ionic basis of transient inward current induced by strophanthidin in cardiac Purkinje fibres. J. Physiol. (Lond.). 281:209-226.

61. Colquhoun, D., E. Neher, H. Reuter, and C. F. Stevens. 1981. Inward current channels activated by intracellular $\mathrm{Ca}$ in cultured cardiac cells. Nature (Lond.). 294:752-754.

62. Mullins, L. J. 1979. The generation of electric currents in cardiac fibers by $\mathrm{Na} / \mathrm{Ca}$ exchange. Am. J. Physiol. 236:C103-C110.

63. Lab, M. J., D. G. Allen, and C. H. Orchard. 1984. The effects of shortening on myoplasmic calcium concentration and on the action potential in mammalian ventricular muscle. Circ. Res. 55:825-829.

64. Kline, R., and M. Morad. 1976. Potassium efflux and accumulation in heart muscle. Evidence from $\mathrm{K}^{+}$electrode experiments. Biophys. J. 16:367-372. 